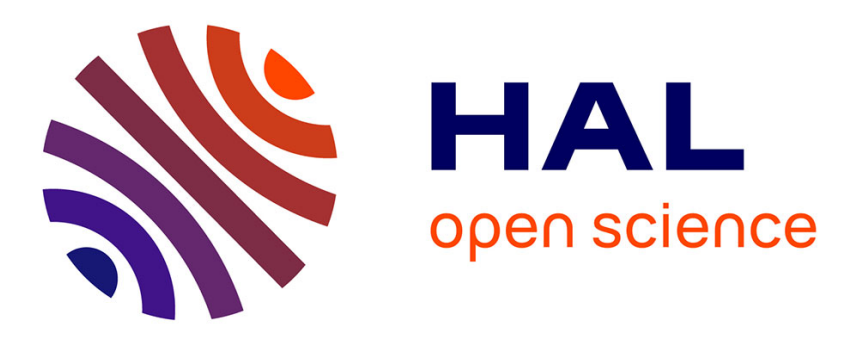

\title{
Machine learning for optimized buildings morphosis
}

Khaoula Raboudi, Abdelkader Ben Saci

\section{To cite this version:}

Khaoula Raboudi, Abdelkader Ben Saci. Machine learning for optimized buildings morphosis. E. Reyes, G. Kembellec, F. Siala-Kallel, L. Sfaxi, M. Ghenima, I. Saleh. DTUC '20: Digital Tools \& Uses Congress, Oct 2020, Virtual Event Tunisia, Proceedings of the 2nd International Conference, Association for Computing Machinery, pp.1-5, 2020, 978-1-4053-7753-9. 10.1145/3423603.3424057 . hal-03258021

\section{HAL Id: hal-03258021 \\ https://hal.science/hal-03258021}

Submitted on 11 Jun 2021

HAL is a multi-disciplinary open access archive for the deposit and dissemination of scientific research documents, whether they are published or not. The documents may come from teaching and research institutions in France or abroad, or from public or private research centers.
L'archive ouverte pluridisciplinaire HAL, est destinée au dépôt et à la diffusion de documents scientifiques de niveau recherche, publiés ou non, émanant des établissements d'enseignement et de recherche français ou étrangers, des laboratoires publics ou privés.

\section{(1) (1) $\$$}

Distributed under a Creative Commons Attribution - NonCommercial - NoDerivatives| 4.0 


\section{Machine learning for optimized buildings morphosis}

\author{
Khaoula Raboudi* \\ khaoula.raboudi@isamm.uma.tn \\ Univ. Manouba, M2a Team \& ISAMM, \\ 1120 Manouba, Tunisia
}

\begin{abstract}
The world is rapidly urbanizing, with an increasing number of new building constructions. This involves increasing the world's energy consumption and its associated greenhouse gas emissions. Computational tools are playing an increasing impact on the architectural design process. Recently, Machine learning (ML) has been applied to building design and has evinced its potential to improve building performance. This paper tries to review the use of ML for the building morphosis. We then forecast the use of machine learning for building optimized morphosis in the early design stage particularly for ensuring summer shading and winter solar access between neighbors.
\end{abstract}

Keywords: Machine learning, building morphosis, solar access, shading.

\section{ACM Reference Format:}

Khaoula Raboudi and Abdelkader Ben Saci. 2020. Machine learning for optimized buildings morphosis. In Proceedings of the 2nd International Digital Tools \& Uses Congress (DTUC '20), October 15-17, 2020, Online, Tunisia. ACM, New York, NY, USA, 5 pages. https://doi.org/10.1145/3423603.3424057

\section{Introduction}

Currently, developing countries are faced to rapid urbanization. The urban population of these countries is projected to double in 2030 and the built-up area to triple [24]. This involves the construction of large volumes of new buildings which not only wastes land, increases energy consumption, but also increases greenhouse gas emissions. To deal with sustainable development challenges, it is important to act on early design decisions, particularly by designing a suitable building morphology. Designing built forms in urban areas is important because the neighborhood building blocks

Permission to make digital or hard copies of all or part of this work for personal or classroom use is granted without fee provided that copies are not made or distributed for profit or commercial advantage and that copies bear this notice and the full citation on the first page. Copyrights for components of this work owned by others than ACM must be honored. Abstracting with credit is permitted. To copy otherwise, or republish, to post on servers or to redistribute to lists, requires prior specific permission and/or a fee. Request permissions from permissions@acm.org.

DTUC '20, October 15-17, 2020, Online, Tunisia

(c) 2020 Association for Computing Machinery. ACM ISBN 978-1-4503-7753-9/20/10 . \$15.00

https://doi.org/10.1145/3423603.3424057

\author{
Abdelkader Ben Saci \\ Univ. Grenoble Alpes, CNRS, School of Architecture, \\ AAU-CRESSON, \\ 38000 Grenoble, France \\ bensaci.a@grenoble.archi.fr
}

are interdependent. Particularly, energy demands over the whole year can be influenced by summer shading effects and winter solar access availability [29-32]. This complex issue still does not have a satisfying solution. Its resolution would be a lever to meet climate and environmental challenges in architectural design.

Tools and methods of computational design used by architects are constantly evolving with the ability to solve complex problems and to account for a high number of variables. From now on they become crucial elements for accompanying the design process, especially with the necessity to cope with environmental challenges. Architecture has always followed computational advances which became fully embedded in the design practice. Recently, Machine Learning (ML), a branch of artificial intelligence, has emerged and it is considered for many authors as an under-way revolution. Many fields are already affected by this new way of solving complex problems, finance, medicine, physics, and computer vision among others. [4] argues that giving on the recent breakthroughs of ML in other areas, it is expectable to see architecture fully adopting it.

Machine learning begins to be applied in the architectural field and has evinced its potential to improve building performance. This paper does not aim to exhaustively tackle every single research. It emphasizes researches related to building morphosis at the early design stage. We try to review how machine learning has been used for this purpose with a focus on used ML models and data generation methods. We then forecast the use of machine learning for optimized building morphosis ${ }^{1}$ in order to ensure summer shading and winter solar access between neighbors.

Study findings can inform the future design with machine learning application to improve building performance in the interest of the occupant's well-being and environment preservation. It can also inspire young researchers in the field of architecture and urbanism to explore multidisciplinary approaches that integrate building science, computing science, and data science.

\footnotetext{
${ }^{1}$ The term morphosis is developed by Ben Saci [5] from the Greek word «morphosis» meaning «action to give form to»
} 


\section{Machine learning for architectural design}

Machine learning applications occur throughout the building design stages improving the design and extending the scope of the design process [37]. The increasing number of research papers using this technique attests that it might have a broad impact on architectural practices. Some authors $[4,8,10]$ have already illustrated ML applications in architectural practice. [4] argues that by coupling ML techniques to the optimization process, it is possible to learn which design parameter yields the better efficiency. ML could help in reducing the running time and the number of variables [4] in architectural design process.We propose to summarize these applications in three different aspects; visualization, simulation and morphosis.

ML has the potential to enhance building visualization and documentation. Current literature used ML techniques to enhance the production of images and 3D models representation and labeling $[1,19]$. In the field of building design, ML is used for plan generation by converting the rasterized floor plan image onto vector graphics representation [20]. We can mention other applications of ML with the ability to produce 3D building representation [40]which can be used for "style transfer" as a primary material for mapping and reproducing a style [41].

The use of ML for simulation gave rise to performancebased design. ML can predict out of precedent simulation results, how the new building would behave. ML has been introduced in building engineering to accelerate complex simulation and predict energy consumption [21, 22, 25], solar radiation [39], daylight availability [3], thermal comfort $[6,15,27]$ or to predict urban heat island phenomena [16]. ML has been used also for the simulation and prediction of other mechanical aspects like material behavior [33]. Predictive models built on machine learning algorithms have shown their effectiveness to the building design community due to their ability to handle complex problems acting like proxies to heavy and time-consuming simulations [3].[25] found that ML based energy consumption forecasting can yield better prediction results when compared with simulation software prediction results. [27] presented a review of ML used to predict thermal comfort. He argues the evidence, arising from several studies and experiments, the potential and benefits of ML algorithms for forecasting occupant thermal comfort compared with traditional methods. The ML based methodology presented in the paper [15] demonstrated that the urban heat island intensity can be predicted quite accurately even when using a limited set of data.

In this paper, we focus on the use of ML for building morphosis which is the creation of building shape design according to different qualitative or quantitative criteria defined beforehand by the designer. The traditional process of architecture design is driven by the designer concepts with the aim to generate suitable building shape. Recent work [35] discusses the application of ML for generating architectural design by the emulation of the human mental reasoning process. This ML based method is used for the building morphosis and is based on qualitative design criteria and personal designer preferences [9, 34, 42], specific architectural styles [26], or functional performance criteria [2]. [34] used ML for the formation of criteria based on the patterns learned from the user's design choices meeting their personal aesthetic, abstract, or qualitative criteria. The selected design solutions are encoded and recorded as a training set for the learning process. The ML based method provided more design possibilities than a human designer and identified additional high performing regions of the solution space based on the user's inexplicit qualitative criteria. [26] explored the ML to generate 3D building massing models relating to a specific urban and stylistic context along with architectural plans and facade designs from a particular stylistic movement in the history of architecture. This research demonstrated that ML could serve as an analytical tool capable of revealing hidden patterns and principals of an architectural style which could be used to produce new specific designs. [2] presented a building function-driven approach to generate new designs. The researcher used ML to evaluate existing designs encoded as graphs and subgraphs and to merge them into a new functional composition.

Machine learning is also used to analyze a reference building design and explore different alternative scenarios, with the aim of identifying the optimal solution. There are researchers that focused on the minimization of construction cost, duration, and Greenhouse gas emissions assessment [11] whereas some emphasized on energy or environmental building performance [7, 12].[11] presented an ML based model to accelerate simulation and multi-objective optimization process by nearly real-time providing fitness evaluation. This method had significantly reduced computing time. [7] used ML as a method for generating all possible urban geometric shapes in order to identify relationships between design parameters and urban performance measures. [12] proposed to use ML to quantify and to learn from the relationships between environmental uncertainties associated with early design decisions. Thus, across this review, we noticed that no ML application was found for optimized building morphosis ensuring summer shading and winter solar access taking into account the complex morphological relationships between neighbors. Although the impacts of built forms on energy performance have been considered in earlier researches, the interactive and complex relationships among neighboring [38] have not been studied with artificial intelligence approaches.

In the next sub-sections, we will present the ML models and the data sourcing methods and discuss their application in early design stage. We finally noted that the potential to 
enhance the building performance by climatic morphosis has not been explored yet by artificial intelligence.

\subsection{Machine learning models}

Regarding the mathematical models used in ML, the most common ones are artificial neural networks (ANNs) $[11,12$, $34,37,42]$. ANNs is an artificial intelligence approach inspired by the biological neural networks. It enables training a dataset in the form of input parameters and corresponding output values, during the learning process [23]. Then, the complicated algorithm design is replaced by learning based on a provided dataset [8]. Nevertheless, ANNs is criticized for its opacity since the correlation of inputs and outcomes is unclear [4].

Deep neural networks (DNNs) are considered as a technique that revolutionized classification and image recognition tasks and even surpassed human vision [26]. [2]presented an application of DNN to extracts designs into essential building blocks based on functional performance criteria. Then generative adversarial networks (GANs) are used to recombine them into new designs. GANs are typically used to generate 2D and 3D designs. [26] used GANs technologies to generate designs based on specific architectural styles. The GANs based method is used to produce unforeseen designs that may have not arisen to human architects. However, GANs may require significant computational resources and time while it can be an affordable technique for architects without any coding experience [26].

Machine learning techniques can be useful to improve optimization process[11]. Clustering techniques such as Kmeans are used for creating a group set of solutions that would be submitted to the costly ML evaluation and optimization process [17].

\subsection{Data sourcing methods}

For the application of machine learning, designers must use large datasets which may not be easy to find as the application of ML is still in its beginning in the architectural field. To deal with this problem, designers can collect data from: publicly available datasets $[28,36]$, genetic algorithms [34, 37], parametric design tools $[7,12,37]$ or building information models [2]. Moreover, the IoT wireless technologies offer a collecting method of the data-related occupant [27] and buildings.

Genetic algorithms are the most widespread type of multiobjective optimization methods. Therefore, they are also becoming a common data generation method in the field of architectural design. They are being explored as a means of developing and altering the "state space" of a design [14].

A combination between ML and parametric generative design enables shape differentiation and search. Parametric design could serve to create design scenarios with building performance simulations. Therefore, designers could use ML to assess large datasets.[7] used reinforcement learning for generating design alternatives and parametric modeling for generating a data set provided from performance simulation.

\section{Discussion}

Despite the growth of machine learning usage, it is still limited in the field of architecture for building morphosis. The architectural practices rely on computer-aided design and building information models. In this paper, we present several alternatives for the use of ML in building design, especially in the building visualization, simulation and morphosis. Across this review, we argue that the ML technique could be an interesting technique for early design exploration and advanced decision making in design optimization, particularly for the building morphosis.

ML could enlarge the solution space with the opportunities to discover new, unexpected spatial forms and combinations [8]. ML enables also higher efficiency in complex shape design and can respond to a vast number of parameters and relationships influencing design. This is done with relatively simple algorithms easily understandable by architects.[8] attests that ML adoption results in more intuitive design tools. We think that the ML technique should be introduced in the field of architectural design and its teaching. We argue that architects should adopt it, master it, and take full advantage of it. Similarly, they shifted in the past from manual drawing to Computer-Aided Design and more recently to parametric design. Now, the future step is data-driven design and artificial intelligence. Regarding this perspective,[18] presents an education framework for teaching ML and facilitating its deployment to architects.

Unfortunately, ML techniques may have some limitations. To run any data-driven ML technique, the designer must provide a large amount of data. A small data set may cause a limitation in the ability to validate predictive capabilities [4]. Recently, open data and Building Information Models (BIM) could help to reduce the data deficit. On the other hand, fully accepting machine learning poses an issue for designers as they fear being replaced during the architectural design process. They might be given the position of a second layer on calculated and generated models. ML currently looks like a 'black box' of the classic design process designated by creativity, inspiration, etc. in the architects' discourse. From this point of view, ML should not disturb much the architects' culture unless it aims to replace a human approach with a more efficient artificial approach.[8] defends the idea that ML should be considered as a collaborator of the human architect rather than its substitute. The idea of the artificial architect is not recent, Friedman already tackled it in the $60 \mathrm{~s}$ with the understanding of that time [13].

This review provides a basis for the ML exploitation in the climatic optimized building morphosis ensuring shading and solar access. Typically, building optimization is addressed by 
deterministic and stochastic methods. It would be interesting to project an optimization method by learning. We must identify which suitable data generation method to adopt and which ML models that support building optimized morphosis.

We notice that parametric tools are widely used by designers for the dataset generation. They are affordable by architects as they allow them to compose algorithms with visual scripting techniques. We hypothesize that parametric tools could offer a technique for morphotic data sourcing with possibilities to generate solutions and to perform simulations based on physical parameters of the environment such as solar radiation. ML could learn morphological parameters from designs imposed by the complexity of interaction between neighbors. Then a generative adversarial network (GANs) could be used to recombine them into new designs.

Finally, future research should compare the presented ML models and experiment the proposed design scenario for optimized building morphosis.

\section{Conclusion}

Designers must consider the effect of their shape design on shading and solar access not only for their building but for surrounding ones as well. Machine learning offers a design method paradigm that could better understand the building geometric properties and complex relationships among neighboring. It could be an interesting tool for optimized building morphosis ensuring summer shading and winter solar access which will greatly help to reduce building's carbon footprint and thus, to minimize the impact of our design on the environment.

\section{References}

[1] Eman Ahmed, Alexandre Saint, Abd El Rahman Shabayek, Kseniya Cherenkova, Rig Das, Gleb Gusev, Djamila Aouada, and Björn E Ottersten. 2018. A Survey on Deep Learning Advances on Different 3D Data Representations. Computer science 1, 1 (2018).

[2] Imdat As, Siddharth Pal, and Prithwish Basu. 2018. Artificial intelligence in architecture: Generating conceptual design via deep learning. International fournal of Architectural Computing 16, 4 (2018), 306-327. https://doi.org/10.1177/1478077118800982

[3] Mohammed Ayoub. 2020. A review on machine learning algorithms to predict daylighting inside buildings. Solar Energy 202 (2020), 249-275. https://doi.org/10.1016/j.solener.2020.03.104

[4] Catarina Belém, Luis Santos, and António Leitão. 2019. On the Impact of Machine Learning. Architecture without Architects?. In Proceedings of the 18th International CAAD Futures Conference, Ji-Hyun Lee (Ed.) South Korea, 148-167.

[5] Abdelkader Ben Saci. 2000. Une théorie générale de l'architecture, morphométrie et modélisation systémique. Thèse de doctorat. Université Jean Moulin, Lyon.

[6] Qian Chai, Huiqin Wang, Yongchao Zhai, and Liu Yang. 2020. Using machine learning algorithms to predict occupants' thermal comfort in naturally ventilated residential buildings. Energy and Buildings 217 (2020), 109937. https://doi.org/10.1016/j.enbuild.2020.109937

[7] Soowon Chang, Nirvik Saha, Daniel Castro-Lacouture, and Perry PeiJu Yang. 2019. Multivariate relationships between campus design parameters and energy performance using reinforcement learning and parametric modeling. Applied Energy 249 (2019), 253-264. https: //doi.org/10.1016/j.apenergy.2019.04.109

[8] Jan Cudzik and Kacper Radziszewski. 2018. Artificial Intelligence Aided Architectural Design. In Proceedings of the 36th eCAADe Conference, A Kepczynska-Walczak and S Bialkowski (Eds.). Poland.

[9] Subhajit Das. 2018. Interactive Artificial Life Based Systems, Augmenting Design Generation and Evaluation by Embedding Expert Opinion - A Human Machine dialogue for form finding.. In Proceedings of the 36th eCAADe Conference, A Kepczynska-Walczak and S Bialkowski (Eds.). Poland, 85-94.

[10] Alfredo Vellido Emil Racec, Stefania Budulan. 2016. Computational Intelligence in architectural and interior design : a state-of-the-art and outlook on the field. Ccia (2016), 108-113.

[11] K Feng, S Chen, and W Lu. 2019. Machine learning based construction simulation and optimization. In Proceedings of Winter Simulation Conference. Institute of Electrical and Electronics Engineers Inc., 20252036. https://doi.org/10.1109/WSC.2018.8632290

[12] Kailun Feng, Weizhuo Lu, and Yaowu Wang. 2019. Assessing environmental performance in early building design stage: An integrated parametric design and machine learning method. Sustainable Cities and Society 50 (2019), 101596. https://doi.org/10.1016/j.scs.2019.101596

[13] Yona Friedman. 1971. Pour l'architecture scientifique (pierre bel ed.). Paris.

[14] John S Gero, Sushil J Louis, and Sourav Kundu. 1994. Evolutionary learning of novel grammars for design improvement. Artificial Intelligence for Engineering Design, Analysis and Manufacturing 8, 2 (1994), 83-94. https://doi.org/10.1017/S089006040000069X

[15] Ali Ghahramani, Guillermo Castro, Simin Ahmadi Karvigh, and Burcin Becerik-Gerber. 2018. Towards unsupervised learning of thermal comfort using infrared thermography. Applied Energy 211 (feb 2018), 41-49. https://doi.org/10.1016/J.APENERGY.2017.11.021

[16] K Gobakis, D Kolokotsa, A Synnefa, M Saliari, K Giannopoulou, and M Santamouris. 2011. Development of a model for urban heat island prediction using neural network techniques. Sustainable Cities and Society 1, 2 (2011), 104-115. https://doi.org/10.1016/j.scs.2011.05.001

[17] Yaochu Jin and Bernhard Sendhoff. 2004. Reducing Fitness Evaluations Using Clustering Techniques and Neural Network Ensembles. In Proceedings of Genetic and Evolutionary Computation - GECCO 2004, Kalyanmoy Deb (Ed.). Springer Berlin Heidelberg, Berlin, Heidelberg, 688-699.

[18] Nariddh Khean and M. Hank Fabbri, Alessandra Haeusler. 2018. Learning Machine Learning as an Architect, How to? - Presenting and evaluating a Grasshopper based platform to teach architecture students machine learning. In Proceedings of the 36th eCAADe Conference, A Kepczynska-Walczak and S Bialkowski (Eds.). Poland, 95-102.

[19] Chen-Hsuan Lin, Chen Kong, and Simon Lucey. 2018. Learning Efficient Point Cloud Generation for Dense 3D Object Reconstruction. In proceedings of the AAAI Conference on Artificial Intelligence. Louisiana.

[20] Chen Liu, Jiajun Wu, Pushmeet Kohli, and Yasutaka Furukawa. 2017. Raster-to-Vector: Revisiting Floorplan Transformation. In Proceedings of the IEEE International Conference on Computer Vision, Vol. 2017Octob. 2214-2222. https://doi.org/10.1109/ICCV.2017.241

[21] Tao Liu, Zehan Tan, Chengliang Xu, Huanxin Chen, and Zhengfei Li. 2020. Study on deep reinforcement learning techniques for building energy consumption forecasting. Energy and Buildings 208 (feb 2020), 109675. https://doi.org/10.1016/J.ENBUILD.2019.109675

[22] Elena Mocanu, Phuong H. Nguyen, Wil L. Kling, and Madeleine Gibescu. 2016. Unsupervised energy prediction in a Smart Grid context using reinforcement cross-building transfer learning. Energy and Buildings 116 (mar 2016), 646-655. https://doi.org/10.1016/J.ENBUILD. 2016.01.030

[23] Mehryar Mohri and Ameet Rostamizadeh, Afshin Talwalkar. 2012. Foundations of Machine Learning. MIT Press, New York. 
[24] Emanuele Naboni and Lisanne Havinga. 2019. Regenerative design in digital practice. A handbook for the built environment. Eurac Research.

[25] Alberto Hernandez Neto and Flávio Augusto Sanzovo Fiorelli. 2008. Comparison between detailed model simulation and artificial neural network for forecasting building energy consumption. Energy and Buildings 40, 12 (2008), 2169-2176. https://doi.org/10.1016/j.enbuild. 2008.06.013

[26] David Newton. 2019. Generative Deep Learning in Architectural Design. Technology Architecture + Design 3, 2 (2019), 176-189. https: //doi.org/10.1080/24751448.2019.1640536

[27] Jack Ngarambe, Geun Young Yun, and Mat Santamouris. 2020. The use of artificial intelligence (AI) methods in the prediction of thermal comfort in buildings: energy implications of AI-based thermal comfort controls. Energy and Buildings (2020), 109807. https://doi.org/10.1016/ J.ENBUILD.2020.109807

[28] Alex Nutkiewicz, Zheng Yang, and Rishee K Jain. 2018. Data-driven Urban Energy Simulation (DUE-S): A framework for integrating engineering simulation and machine learning methods in a multi-scale urban energy modeling workflow. Applied Energy 225 (2018), 11761189. https://doi.org/10.1016/j.apenergy.2018.05.023

[29] Khaoula Raboudi. 2017. Générateur morphologique de règles urbaines de contrôle solaire. Thèse de doctorat. Université de Carthage.

[30] Khaoula Raboudi and Abdelkader Ben Saci. 2013. A morphological generator of urban rules of solar control. In Sustainable Architecture for a Renewable Future: Proceedings of the 29th International Conference on Passive and Low Energy Architecture (PLEA 2013). Munich.

[31] Khaoula Raboudi and Abdelkader Ben Saci. 2014. Génération de volumes de contrôle solaire. In Interaction(s) des maquettes numériques. Presses universitaires de Nancy, Luxembourg, 221-231.

[32] Khaoula Raboudi and Abdelkader Ben Saci. 2018. Conditions of Satisfaction of the Solar Control Box Constraints. Fournal of Civil Engineering and Architecture 12, 10 (2018), 685-693. https://doi.org/10.17265/ 1934-7359/2018.10.001

[33] Denise Reimann, Kapil Nidadavolu, Hamad ul Hassan, Napat Vajragupta, Tobias Glasmachers, Philipp Junker, and Alexander Hartmaier. 2019. Modeling Macroscopic Material Behavior With Machine Learning Algorithms Trained by Micromechanical Simulations. Frontiers in Materials 6 (2019), 181. https://doi.org/10.3389/fmats.2019. 00181

[34] Christian Sjoberg, Christopher Beorkrem, and Jefferson Ellinger. 2017. Emergent Syntax: Machine Learning for the Curation of Design Solution Space.

[35] K Steinfeld. 2017. Dreams may come. In Proceedings of the 37th annual conference of the Association for Computer Aided Design in Architecture, T Nagakura (Ed.). Cambridge, 590-599.

[36] F Sun, M Liu, Y Wang, H Wang, and Y Che. 2020. The effects of 3D architectural patterns on the urban surface temperature at a neighborhood scale: Relative contributions and marginal effects. fournal of Cleaner Production 258 (2020). https://doi.org/10.1016/j.jclepro.2020.120706

[37] Martin Tamke, Paul Nicholas, and Mateusz Zwierzycki. 2018. Machine learning for architectural design: Practices and infrastructure. International fournal of Architectural Computing 16, 2 (2018), 123-143. https://doi.org/10.1177/1478077118778580

[38] Laila Turki Koubaa, Khaoula Raboudi, and Abdelkader Ben Saci. 2018. Stratégies de prospect du droit solaire par l'immersion. In Immersion émersion et conception de projets architecturaux et urbains (edp scienc ed.), Thomas Leduc and Françis Miguet (Eds.). Nantes. https://doi.org/ 10.1051/shsconf/20184701014

[39] Aristotelis Vartholomaios. 2019. A machine learning approach to modelling solar irradiation of urban and terrain 3D models. Computers Environment and Urban Systems 78 (2019).
[40] Andreas Wichmann, Amgad Agoub, and Martin Kada. 2018. ROOFN3D: Deep learning training data for 3D building reconstruction. International Archives of the Photogrammetry, Remote Sensing and Spatial Information Sciences - ISPRS Archives 42, 2 (2018), 1191-1198. https://doi.org/10.5194/isprs-archives-XLII-2-1191-2018

[41] Hang Zhang. 2019. 3D Architectural Form Style Transfer through Machine Learning. In Porceedings of the 25th International Conference of the Association for Computer-Aided Architectural Design Research. Bangkok. https://doi.org/10.13140/RG.2.2.16791.52645

[42] Hao Zheng. 2020. Form Finding and Evaluating Through Machine Learning: The Prediction of Personal Design Preference in Polyhedral Structures. In Proceedings of the 2019 DigitalFUTURES. https://doi. org/10.1007/978-981-13-8153-9 